Journal of

Synchrotron

\section{Radiation}

ISSN 0909-0495

Received 2 March 2010

Accepted 8 April 2010

\title{
Simultaneous measurement of X-ray specular reflection and off-specular diffuse scattering from liquid surfaces using a two-dimensional pixel array detector: the liquid-interface reflectometer of BL37XU at SPring-8
}

\author{
Yohko F. Yano, ${ }^{a *}$ Tomoya Uruga, ${ }^{\mathrm{b}}$ Hajime Tanida, ${ }^{\mathrm{b}}$ Hidenori Toyokawa, \\ Yasuko Terada ${ }^{b}$ and Hironari Yamada ${ }^{c}$ \\ ${ }^{\text {a }}$ Research Organization of Science and Engineering, Ritsumeikan University, Shiga, Japan, \\ bJapan Synchrotron Radiation Research Institute, Hyogo, Japan, and ' Department of Photonics, \\ Ritsumeikan University, Shiga, Japan. E-mail: y-yano@fc.ritsumei.ac.jp
}

\begin{abstract}
An X-ray reflectometer for simultaneous measurement of specular and offspecular reflection of liquid surfaces is described. The reflectometer, equipped with a two-dimensional single $\mathrm{X}$-ray photon-counting pixel array detector obtained the full range of X-ray specular and off-specular reflections in an extremely short time $(1 \mathrm{~s})$. Both the specular and off-specular reflection of water exhibited good agreement with the predicted capillary-wave theory within the appropriate instrumental resolution. The approach is also demonstrated on an aqueous solution of 1-dodecyl-3-methylimidazolium chloride. The monolayer in which the dodecyl chain faces upwards and the $\mathrm{Cl}^{-}$anion locates next to the imidazolium ring formed on the water surface was found to be laterally homogeneous. The use of a pixel array detector will be particularly powerful for in situ measurements to investigate both out-of-plane and in-plane structures simultaneously, not only for liquid surfaces but also for other thin films.
\end{abstract}

Keywords: X-ray reflectivity; off-specular diffuse scattering; PILATUS; air/water interface; ionic liquids.

\section{Introduction}

Over the past 20 years, X-ray reflectivity measurements using synchrotron X-ray sources have been one of the most powerful tools for investigating the surface phenomena of liquids (Daillant \& Gibaud, 1999; Tolan, 1999). Such techniques allow the density profile to be measured along the surface normal on a sub-angstrom spatial resolution. By carefully examining diffuse scattering in the vicinity of specular reflection peaks, structural information parallel to the surface can also be obtained.

The surface morphology of liquid surfaces is governed by thermally excited capillary waves with molecular-size amplitudes and micrometer-order wavelengths (Buff et al., 1965). Sinha et al. have theoretically demonstrated that diffuse scattering from liquid surfaces diverges algebraically at the specular reflection angle, creating large tails that extend into the surrounding off-specular region (Sinha et al., 1988). This theoretical prediction has been verified experimentally for simple molecular liquids (Braslau et al., 1988; Schwartz et al., 1990; Sanyal et al., 1991; Pershan, 2000; Shpyrko et al., 2004) and liquid metals (Pershan, 2000; Shpyrko et al., 2003, 2004;
Tostmann et al., 1999). In contrast, scattering that exceeds the predicted capillary contribution has been observed for Langmuir monolayers on a water surface, and provides lateral structural information on the monolayers (Li et al., 1995; Fukuto et al., 1998, 1999, 2004). However, X-ray off-specular diffuse scattering intensities are always difficult to evaluate correctly since the instrumental-resolution dependent weak signals are hidden in large background intensities arising from the signals of other sources. Such measurements, therefore, are usually conducted independently of X-ray reflectivity measurements with a high angle-resolution system.

Previously, we have reported a new liquid-interface reflectometer developed at SPring-8 (Yano et al., 2007, 2009a). This reflectometer is equipped with a two-dimensional single $\mathrm{X}$-ray photon-counting pixel array detector, PILATUS $100 \mathrm{~K}$ (Dectris, Switzerland), and can achieve an X-ray reflectivity of almost $10^{-9}$ with an accumulation time of only $1 \mathrm{~s}$ at each angle, exhibiting enormous potential for rapid measurements. Time-resolved measurements with a time resolution of $3 \mathrm{~min}$ were performed for the adsorption of a globular protein on an air/water interface (Yano et al., 2009b,c). The PILATUS detector also offers many other advantages such as the ability 
to collect area images, a wide dynamic range of $10^{6}$, an excellent point-spread function of one pixel, zero dark noise and the option to set an energy threshold for background suppression (Broennimann et al., 2006; Kraft et al., 2009), enabling measurement of the weak diffuse scattering signals that lie very close to the much stronger specular reflection.

In the present study we exploit the advantages of the twodimensional pixel array detector to investigate both the X-ray specular reflection and the off-specular diffuse scattering intensities from two different liquid surfaces, a pure water surface and a surfactant monolayer on water. By comparing our results with the well established capillary-wave theory, we show that the reflectometer can accurately measure the X-ray specular reflection and off-specular diffuse scattering intensities in an extremely short time.

\section{Scattering from liquid surfaces}

\subsection{Geometry}

The scattering geometry is illustrated in Fig. 1. The incident wavevector $\mathbf{k}_{\text {in }}$ strikes the liquid surface at an incident angle $\alpha$. The scattered X-rays are characterized by the output wavevector $\mathbf{k}_{\text {out }}$, which makes an angle $\beta$ with the surface and an angle $2 \theta$ with the plane of incidence. The Cartesian components of the wavevector transfer $\mathbf{q}=\mathbf{k}_{\text {out }}-\mathbf{k}_{\text {in }}$ are defined as

$$
\begin{aligned}
& q_{z}=k[\sin (\alpha)+\sin (\beta)], \\
& q_{x}=k \cos (\beta) \sin (2 \theta), \\
& q_{y}=k[\cos (\beta) \cos (2 \theta)-\cos (\alpha)],
\end{aligned}
$$

where $k=2 \pi / \lambda$.

\subsection{X-ray specular reflectivity}

In the specular reflection condition, the reflectivity is measured as a function of $q_{z}$ when $q_{x}=q_{y}=0$, or equivalently $\beta=\alpha$ and $2 \theta=0$.

The X-ray specular reflectivity for liquid surfaces is described by the Born approximation (Pershan, 2000, 2009),

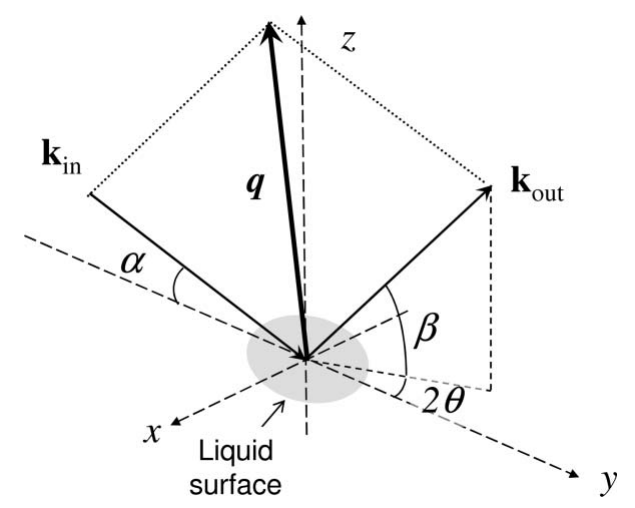

Figure 1

$\mathrm{X}$-ray scattering geometry. Incident wavevector $\mathbf{k}_{\text {in }}$ strikes the liquid surface at an incident angle $\alpha$. The scattered X-rays are characterized by the output wavevector $\mathbf{k}_{\text {out }}$, which makes an angle $\beta$ with the surface and an angle $2 \theta$ with the plane of incidence.

$$
R\left(q_{z}\right)=R_{\mathrm{F}}\left|\Phi\left(q_{z}\right)\right|^{2} C W\left(q_{z}, T, \gamma\right),
$$

where $R_{\mathrm{F}}$ is the Fresnel reflectivity for an ideally flat interface and $\left|\Phi\left(q_{z}\right)\right|^{2}$ is the intrinsic structure factor normal to the surface, which is expressed as

$$
\left|\Phi\left(q_{z}\right)\right|^{2}=\left|\frac{1}{\rho^{\text {Bulk }}} \int \mathrm{d} z \frac{\partial(\rho(z)\rangle_{x y}}{\partial z} \exp \left(i q_{z} z\right)\right|^{2},
$$

where $\langle\rho(z)\rangle_{x y}$ is the lateral average electron density profile. $C W\left(q_{z}, T, \gamma\right)$ is the surface roughness term due to capillary waves and is given by

$$
C W\left(q_{z}, T, \gamma\right)=\exp \left(-\sigma_{\mathrm{cw}}^{2} q_{z}^{2}\right)
$$

with

$$
\sigma_{c w}^{2}=\left(\frac{k_{\mathrm{B}} T}{2 \pi \gamma}\right) \ln \left(\frac{q_{\mathrm{max}}}{\Delta q_{\mathrm{res}}}\right),
$$

where $\gamma$ is the surface tension and $q_{\max }$ is the upper cut-off for capillary contributions, which is determined by the condition in which the number of capillary-wave modes is of the order of the number of molecules per unit surface area; this is equivalent to fixing $q_{\max } \simeq 2 \pi / d$, where $d$ is of the order of the intermolecular distance. $\Delta q_{\text {res }}$ is the instrumental resolution, usually defined by the detector slits (Braslau et al., 1988; Pershan, 2009).

\subsection{X-ray off-specular diffuse scattering}

In off-specular diffuse scattering the scattering intensity is measured under non-specular conditions, i.e. $\alpha \neq \beta$ or equivalently $q_{x}, q_{y} \neq 0$. A $\beta$-scan method, in which the scattered intensity is measured as a function of $\beta$ at constant $\alpha$, has typically been conducted independently of X-ray reflectivity measurements. In the present study we used the reflection profile, normalized to the peak intensity of the reflection profile at an incident angle $\alpha$ of $0.01^{\circ}$ (assumed to be equivalent to the incident beam intensity $I_{0}$ ), as the off-specular diffuse scattering intensity.

The normalized intensity $I / I_{0}$ is generally equal to the convolution of the differential cross section $\mathrm{d} \sigma / \mathrm{d} \Omega$ with an appropriate instrumental resolution function $\Xi$ (Fukuto et al., 1999),

$$
\begin{gathered}
\frac{I(\mathbf{q})}{I_{0}}=\int \frac{\mathrm{d}^{2} \mathbf{q}_{x y}^{\prime}}{k^{2} \sin (\beta)} \Xi_{q}\left(\mathbf{q}_{x y}-\mathbf{q}_{x y}^{\prime}\right) \frac{1}{A_{0}} \frac{\mathrm{d} \sigma}{\mathrm{d} \Omega}\left(\mathbf{q}_{x y}^{\prime}\right), \\
\Xi_{q}\left(\delta q_{x}, \delta q_{y}\right)= \begin{cases}1 & \text { if }\left|\delta q_{x}\right| \leq \Delta q_{x} / 2,\left|\delta q_{y}\right| \leq \Delta q_{y} / 2, \\
0 & \text { otherwise. }\end{cases}
\end{gathered}
$$

The differential cross section for a homogeneous liquid surface is described by the capillary-wave model (Pershan, 2000,2009 ) and can be written as

$$
\begin{aligned}
\frac{1}{A_{0}}\left(\frac{\mathrm{d} \sigma}{\mathrm{d} \Omega}\right)_{\mathrm{hmg}} \simeq & \frac{1}{16 \pi^{2}}\left(\frac{q_{\mathrm{c}}}{2}\right)^{4} \frac{T_{\mathrm{F}}(\alpha) T_{\mathrm{F}}(\beta)}{q_{z}^{2} \sin (\alpha)} \\
& \times\left|\Phi\left(q_{z}\right)\right|^{2} \frac{2 \pi \eta}{q_{x y}^{2}}\left(\frac{q_{x y}}{q_{\max }}\right)^{\eta},
\end{aligned}
$$


(a) Top view

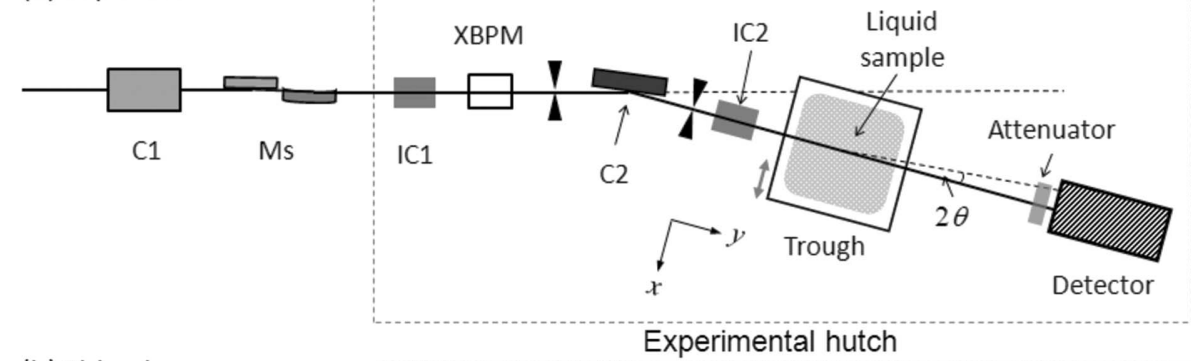

(b) Side view

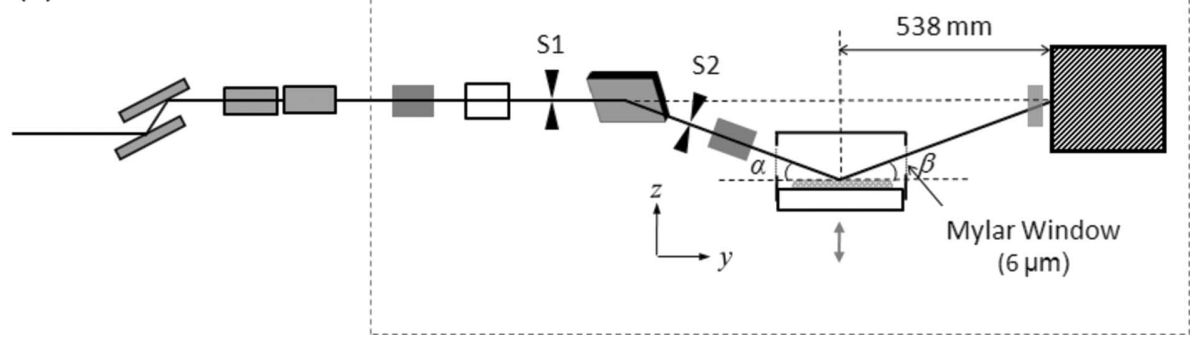

Figure 2

Schematic view of the liquid-interface reflectometer at the SPring-8 BL37XU beamline. C1: Si 111 double-crystal monochromator; $\mathrm{C} 2$ : Ge 111 deflecting-crystal monochromator; Ms: two horizontal deflecting mirrors; IC1 and IC2: ion chambers; XBPM: X-ray beam-position monitor for MOSTAB; $\mathrm{S} 1$ and $\mathrm{S} 2$ : slit systems.

for $\eta=\left(k_{\mathrm{B}} T / 2 \pi \gamma\right) q_{z}^{2}<2$, where $A_{0}$ is the cross-sectional area of the incident beam, $q_{\mathrm{c}}$ is the critical vector, and $T_{\mathrm{F}}(\alpha)$ is the Fresnel transmission factor (Daillant \& Gibaud, 1999; Tolan, 1999). The scattering from a homogeneous liquid surface is described by the characteristic power law, $1 / q_{x y}^{2-\eta}$, of capillarywave thermal diffuse scattering.

\section{Experimental details}

\subsection{Liquid interface reflectometer using a pixel array detector}

The liquid-interface reflectometer (Yano et al., 2007, 2009a) is installed at the undulator beamline BL37XU at SPring-8 (Terada et al., 2004). The experimental set-up is schematically given in Fig. 2.

An X-ray energy of $15 \mathrm{keV}(\lambda=0.826 \AA)$ was selected for the $\mathrm{X}$-ray reflectivity measurements. Two horizontal deflecting Rh-coated mirrors eliminated higher harmonics and focused the X-ray beam to a spot about $200 \mu \mathrm{m}$ in the horizontal direction at the detector position. The design of the liquidinterface reflectometer (Yano et al., 2007, 2009a) is similar to the surface-horizontal spectrometers at other synchrotron facilities described previously (Als-Nielsen et al., 1982; Schlossman et al., 1997). A monochromator stabilization (MOSTAB) system stabilized the X-ray beam intensity by tuning of the Bragg angle of the first crystal of the monochromator (Kudo \& Tanida, 2007). The X-ray beam deflected downwards by the Ge 111 crystal (C2) was collimated by the second slit (S2), which runs $50 \mu \mathrm{m}$ vertically and $500 \mu \mathrm{m}$ horizontally. The beam flux incident on the sample was about $10^{12}$ photons s$^{-1}$. A trough made of aluminium with a teflon coating $\left(100 \mathrm{~cm}^{2}, 3 \mathrm{~mm}\right.$ deep) was located halfway between the deflection crystal $\mathrm{C} 2$ and the detector. A single X-ray photoncounting pixel detector, the PILATUS 100K (Broennimann et al., 2006; Kraft et al., 2009), with an area of $487 \times 195$ pixels $\left(172 \mu \mathrm{m} \mathrm{pixel}^{-1}\right)$ was located $538 \mathrm{~mm}$ from the center of the trough. The integration time at each incident angle was $1 \mathrm{~s}$. Each pixel of PILATUS contains a charge-sensitive amplifier, a shaper amplifier, a single-level discriminator and a 20-bit counter. The energy threshold of each discriminator was set to $7.5 \mathrm{keV}$ to suppress the lowenergy background. The counting-rate capability depends on the operation mode of the storage ring at SPring-8, which is about $100 \mathrm{~ns}$ in the multi-bunch operation mode. This condition allows us to detect up to $10^{6}$ counts pixel ${ }^{-1} \mathrm{~s}^{-1}$ within the dead time of $10 \%$. A set of aluminium sheets of thickness in the range 1-6 $\mathrm{mm}$ was employed as an attenuator at the incident angle $\alpha<0.9^{\circ}$ to prevent the counting rate exceeding $10^{5}$ counts pixel $^{-1} \mathrm{~s}^{-1}$. The seven axes of the motor drivers as shown in Fig. 3 were synchronized to start by a VME pulse motor controller (Furukawa \& Matsumoto, 2004) to shorten the travel time to about $2 \mathrm{~s}$ for the change in incident angle.

The liquid samples were contained in the trough controlled at $298 \mathrm{~K}$. The trough was covered with an acrylic hood with $6 \mu \mathrm{m}$-thick mylar windows for the X-ray measurements to suppress liquid evaporation. The accumulation time at each incident angle was $1 \mathrm{~s}$ for $\alpha<2.0^{\circ}$ and $10 \mathrm{~s}$ for $2.0^{\circ}<\alpha<3.0^{\circ}$, meaning the overall time required to measure the reflection intensities for 81 incident angles was $5 \mathrm{~min}$.

\subsection{Liquid samples}

X-ray reflectivity measurements of a water surface and a monolayer of 1-dodecyl-3-methylimidazolium chloride on

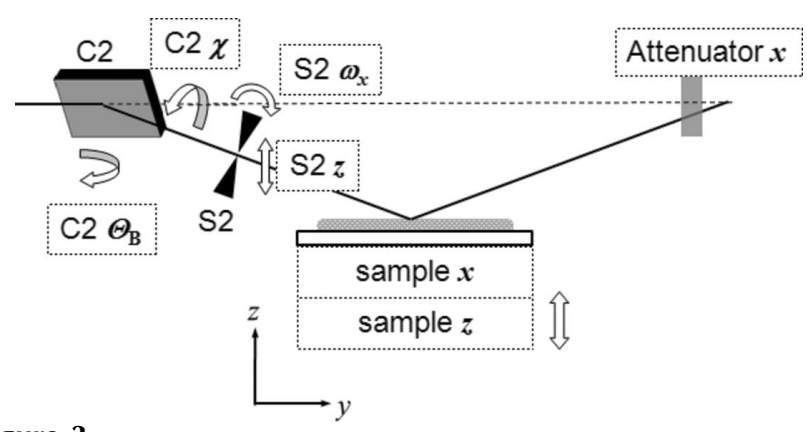

Figure 3

Description of the motor drivers. The deflecting crystal (C2) is rotated by an angle described by $\chi$ and $\Theta_{\mathrm{B}}$. The slit (S2) is adjusted by $\omega_{x}$ and $z$. The liquid sample is set on the two translation stages $x$ and $z$. A set of aluminium sheets of thickness $1-6 \mathrm{~mm}$ is set on the translation stage as an attenuator. 
water were performed. The 1-alkyl-3-methylimidazolium salts (hereafter denoted as $\left[\mathrm{C}_{n} \mathrm{mim}\right] \mathrm{Cl}$, where $n$ is the alkyl chain length) are ionic liquids which have low melting temperatures owing to the low symmetry of the organic cations, which acts to reduce the lattice energy (Seddon, 1997). The $\left[\mathrm{C}_{12} \mathrm{mim}\right] \mathrm{Cl}$ salt is a solid at room temperature and acts analogously to cationic surfactants owing to its long alkyl chain. The selfaggregation behavior has been studied previously (Blesic et al., 2007). In the present study we investigated the surface structure of an aqueous $\left[\mathrm{C}_{12} \mathrm{mim}\right] \mathrm{Cl}$ solution at the critical micelle concentration $\left(x_{\mathrm{cmc}}=0.02 \mathrm{~mol} \mathrm{dm}^{-3}\right)$.

\subsection{Data treatments}

Fig. 4 shows typical images around the reflection peaks detected by PILATUS. For $\alpha=0.7^{\circ}$ the reflection intensity was detected through an aluminium attenuator of thickness $1 \mathrm{~mm}$ placed $10 \mathrm{~mm}$ from the detector, while at $\alpha \geq 0.9^{\circ}$ it was

(a) $\alpha=0.7^{\circ}$ (with Al $1 \mathrm{~mm}$ )
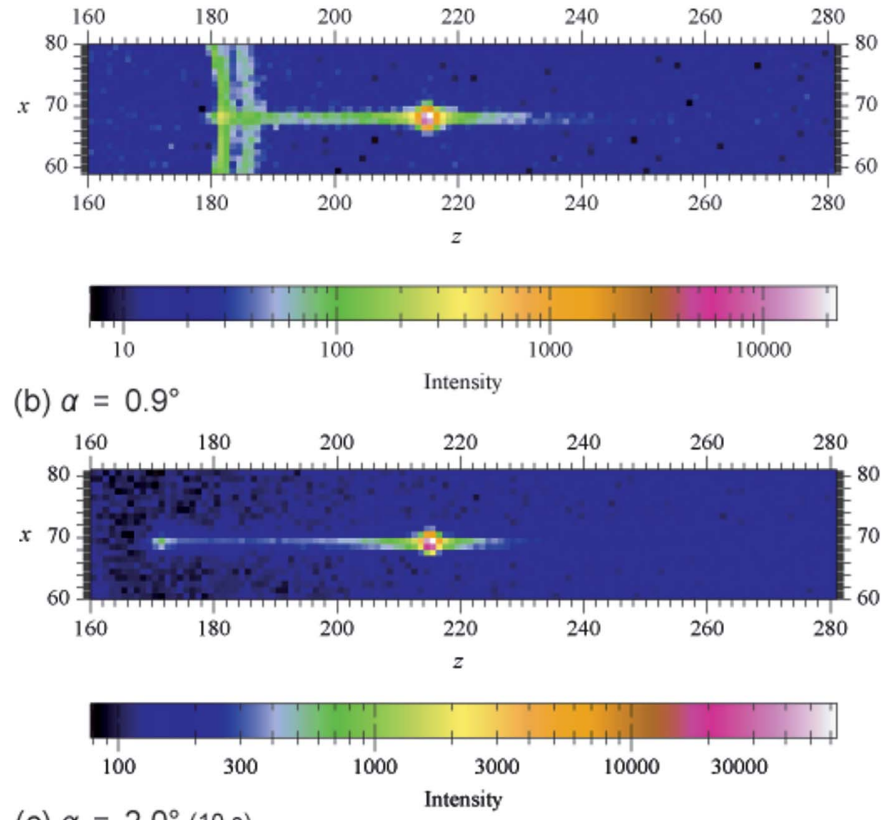

(c) $\alpha=2.0^{\circ}(10 \mathrm{~s})$
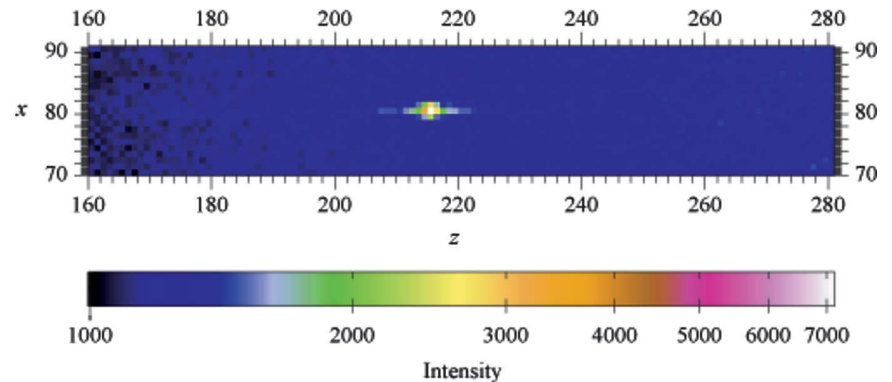

Figure 4

Enlargement of an X-ray specular reflection image of water obtained on PILATUS. The units of the $x$ - and $z$-axes are pixels. Measurement conditions: (a) $\alpha=0.7^{\circ}$, integration time of $1 \mathrm{~s}$ with a $1 \mathrm{~mm} \mathrm{Al}$ attenuator; (b) $\alpha=0.9^{\circ}$, integration time of $1 \mathrm{~s}$ without attenuator; (c) $\alpha=2.0^{\circ}$, integration time of $10 \mathrm{~s}$ without attenuator. Each square corresponds to a pixel with dimensions of $172 \mu \mathrm{m} \times 172 \mu \mathrm{m}$. detected directly. The reflection peaks located at around $(x, z) \simeq(70,216)$ in pixels for all of the incident angles have long tails in the $z$-direction caused by diffuse scattering from the capillary waves. A small peak, known as a Yoneda wing (Yoneda, 1963), is clearly visible at $z \simeq 170$ for $\alpha=0.9^{\circ}$ as shown in Fig. 4(b); for $\alpha=0.7^{\circ}$, shown in Fig. 4(a), the DebyeScherrer rings of the aluminium attenuator observed at around $z \simeq 180-190$ almost overwhelm the Yoneda wing.

Since this reflection peak was detected over five pixels in the $x$-direction, we considered the intensity measured five pixels from the center of the peak to be background. The background intensity of 100-150 counts pixel ${ }^{-1} \mathrm{~s}^{-1}$ is almost independent of the X-ray incident angle. A reflection profile $I(z)$ was obtained by summing the signal intensities and subtracting the background as

$$
\frac{I(z)}{I_{0}}=\frac{1}{I_{0}}\left[\sum_{|x| \leq 2} I(z, x)-\frac{5}{6} \sum_{5 \leq|x| \leq 7} I(z, x)\right] .
$$

The factor of $5 / 6$ is the ratio between the five pixels of the signals and the six pixels of the background. The total signal intensity corresponding to the first term in (7) and the background corresponding to the second term are shown in Fig. 5. In the case of $\alpha=0.7^{\circ}$, the intensity is reduced to about a tenth

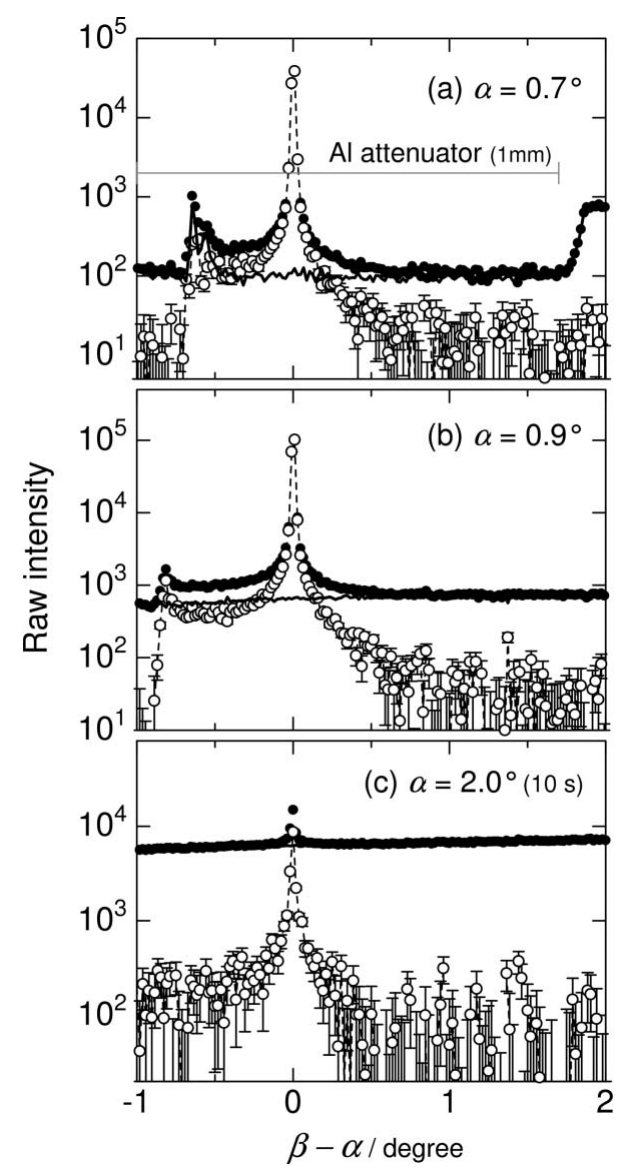

Figure 5

Integrated signal (open circles), integrated background (solid curves) and integrated signal plus background (closed symbols) for the reflection images shown in Fig. 4. 
by the $1 \mathrm{~mm}$-thick aluminium attenuator placed at $\beta-\alpha<2^{\circ}$. After the background subtraction, reflection profiles spanning a range of four orders of magnitudes were obtained. Since the diffuse scattering intensity decreases with the angle $\beta$, in contrast with the $\beta$-independent background intensity, the signal-to-background ratio becomes less than $10 \%$ in the region $\beta-\alpha>1^{\circ}$.

\section{Results and discussions}

\subsection{X-ray specular reflectivity}

Fig. 6(a) shows X-ray reflectivity profiles of the liquid surfaces examined. The reflectivity is calculated by summing the reflection profiles (see Fig. 5) around the peak region of $n$ pixels ( 7 pixels for water and 13 pixels for the aqueous $\left[\mathrm{C}_{12} \mathrm{mim}\right] \mathrm{Cl}$ solution) and normalizing this value to the intensity of the incident beam, which was monitored using an ionization chamber. The instrumental resolution, $\Delta q_{\text {res }}$ in equation (4), is defined for simplicity as the resolution in the $q_{y}$ direction, i.e. $\Delta q_{y} \simeq k \sin (\beta) \Delta \beta$, where $\Delta \beta$ is related to the number of pixels summed to calculate the reflectivity as $n$ pixels $\times 0.172 \mathrm{~mm}$ pixel $^{-1}(538 \mathrm{~mm})^{-1}$. In the higher $q_{z}$ range, however, $\Delta q_{x}$ should be taken into account in addition to $\Delta q_{\text {res }}$ to describe the capillary effect correctly (Pershan, 2009). The predicted capillary-wave model for water calculated using equations (2)-(4) with the parameters $\gamma=72.0 \mathrm{mN} \mathrm{m}^{-1}$, $q_{\max } \simeq 2 \pi / d=2 \pi / 2.8 \AA^{-1}$ and $\left|\Phi\left(q_{z}\right)\right|^{2}=1$ (Braslau et al., 1988$)$ shows good agreement with the observed reflectivity in the present $q_{z}$ range.

In the case of the aqueous $\left[\mathrm{C}_{12} \mathrm{mim}\right] \mathrm{Cl}$ solution, the observed data, divided by the capillary-wave term $C W\left(q_{z}, T\right.$, $\gamma$ ) using parameters $\gamma=40 \mathrm{mN} \mathrm{m}^{-1}$ and $q_{\max } \simeq 2 \pi / d=2 \pi /$ $12 \AA^{-1}$, were fitted using a two-box model. The refined electron density profile is shown in Fig. 6(b). Based on the electron densities of the close-packed structures $\left[0.32 \mathrm{e}^{-3}\right.$ for the

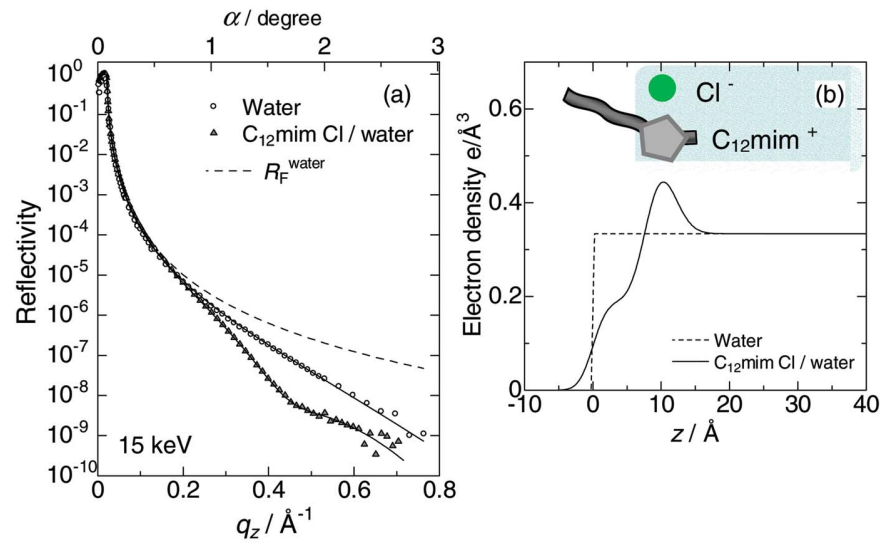

Figure 6

(a) X-ray reflectivity of water (circles) and aqueous $\left[\mathrm{C}_{12}\right.$ mim] $\mathrm{Cl}$ solution (triangles). The solid curve for water is the theoretical prediction calculated using equations (2)-(4) with $R_{\mathrm{F}}$ denoted by the dashed curve. The data for the aqueous $\left[\mathrm{C}_{12} \mathrm{mim}\right] \mathrm{Cl}$ solution were fitted using a two-box model based on the dodecyl chain layer and the $\mathrm{Cl}^{-}$anion layer. (b) Electron density profiles corresponding to the data fits. The inset shows a schematic of the $\left[\mathrm{C}_{12} \mathrm{mim}\right] \mathrm{Cl}$ monolayer arrangement. alkanes, $0.33 \mathrm{e}^{-3}$ for the imidazolium ring and $0.73 \mathrm{e} \AA^{-3}$ for the $\mathrm{Cl}^{-}$anion (Solutskin et al., 2005; Larriba et al., 2009)], the $\left[\mathrm{C}_{12} \mathrm{mim}\right] \mathrm{Cl}$ molecules are considered to form a monolayer in which the dodecyl chain faces upwards and the $\mathrm{Cl}^{-}$anion locates next to the imidazolium ring at a depth of $10 \AA$.

\subsection{X-ray off-specular diffuse scattering}

Fig. 7 shows the normalized reflection profile of X-rays incident at angles of $\alpha=0.9$ and $1.2^{\circ}$ detected in $1 \mathrm{~s}$ without the $\mathrm{Al}$ attenuator. The circles show the results for water, while the triangles show those for the aqueous $\left[\mathrm{C}_{12} \mathrm{mim}\right] \mathrm{Cl}$ solution. The central peaks correspond to specular reflections. The dashed and solid curves in Fig. 7 correspond to the theoretically predicted intensities for the capillary-wave model calculated using equations (5) and (6). Since the differential cross section in (6) diverges at $q_{x y}=0$, we calculated the diffuse scattering intensities in the region $q_{x y}>10^{-5} \AA^{-1}$. The calculated diffuse scattering intensities exhibit good agreement with the observed intensities, especially around the specular region, having instrumental resolutions of $\Delta q_{x} \simeq$ $k \Delta(2 \theta)$ and $\Delta q_{y} \simeq k \sin (\beta) \Delta \beta$, with $\Delta(2 \theta) \simeq 2.3$ pixels $\times$ $0.172 \mathrm{~mm} \operatorname{pixel}^{-1}(538 \mathrm{~mm})^{-1}$ and $\Delta \beta \simeq 3.0$ pixels $\times$ $0.172 \mathrm{~mm}$ pixel $^{-1}(538 \mathrm{~mm})^{-1}$, which are 1.5 times larger than the FWHMs of the reflection profile at the incident angle $\alpha$ of $0.01^{\circ}$. This demonstrates the benefit of the excellent pointspread function of the PILATUS detector. The present result also suggests that the $\left[\mathrm{C}_{12} \mathrm{mim}\right] \mathrm{Cl}$ monolayer is not a laterally inhomogeneous layer, which would lead to excess scattering above the predicted capillary-wave fluctuations.

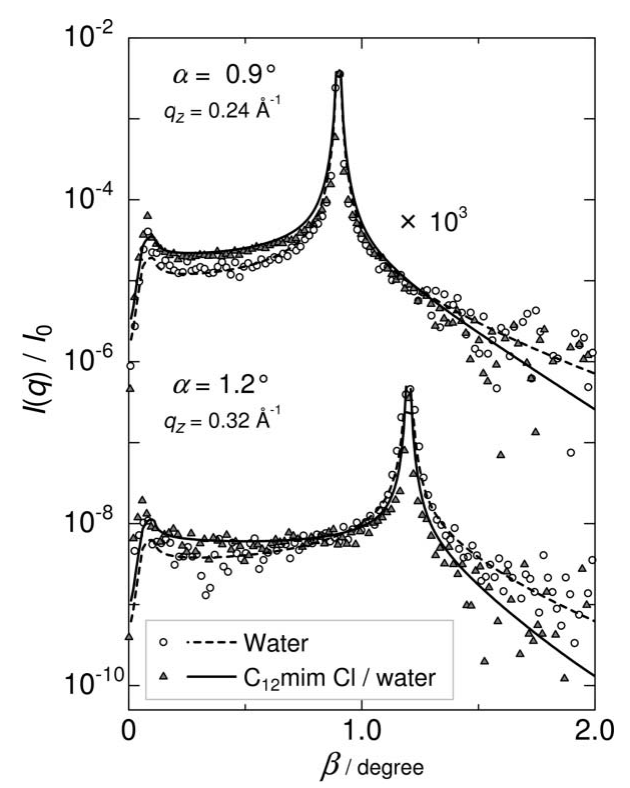

Figure 7

Reflection profiles of liquid surfaces for X-ray incident angles $\alpha=0.9$ and $1.2^{\circ}$. The solid and dashed curves represent the theoretical predictions based on the capillary-wave model and are calculated using equations (5) and (6). Both of the observed intensities for water (circles) and the aqueous $\left[\mathrm{C}_{12} \mathrm{mim}\right] \mathrm{Cl}$ solution (triangles) exhibit good agreement with those for the capillary-wave model. 


\section{Summary}

We performed X-ray reflection measurements of a water surface using a recently developed liquid-interface reflectometer at SPring-8. The reflectometer, equipped with a twodimensional single X-ray photon-counting pixel array detector, PILATUS, obtained an X-ray reflectivity curve towards $10^{-10}$ in $5 \mathrm{~min}$. The detector was also found to be effective in analyzing the weak off-specular diffuse scattering signals in the vicinity of the specular reflection peaks. The results exhibited good agreement with theoretical predictions for ratios of the signal-to-background intensity of larger than $10 \%$. X-ray reflection measurement of an aqueous solution of 1-dodecyl-3-methylimidazolium chloride at the critical micelle concentration was also demonstrated. Based on the specular reflection, 1-dodecyl-3-methylimidazolium chloride was considered to form a monolayer in which the dodecyl chain faces upwards and the $\mathrm{Cl}^{-}$anion locates next to the imidazolium ring on the water surface. The off-specular diffuse scattering indicated that the monolayer was laterally homogeneous because the observed intensity shows good agreement with that predicted by the capillary-wave model.

The present reflectometer accurately obtains the full range of X-ray specular and off-specular reflections in an extremely short time (1s), having enormous potential to determine the out-of-plane and in-plane structures at an interface simultaneously. The methodology developed in the present work can be applied not only for liquid surfaces but also for other thin films.

We are grateful to K. Iimura (Utsunomiya University), N. Ikeda (Fukuoka Women's University) and T. Takiue (Kyushu University) who made great efforts in developing the present liquid-interface reflectometer at SPring-8, and to P. S. Pershan (Harvard University) for helpful discussions and suggestions about the data analysis. The synchrotron radiation experiments were performed at BL37XU at SPring-8 facility with the approval of the Japan Synchrotron Radiation Research Institute (JASRI) (Proposal Nos. 2009A1686, 2006A1124, 2006B1751, 2007A1737, 2007B1212, 2007B1353). The present study was partly supported by a Grant in Aid for Scientific Research (No. 18045029) in Priority Area 'Science of Ionic Liquids' from the Ministry of Education, Culture, Sports, Science and Technology of Japan.

\section{References}

Als-Nielsen, J., Christensen, F. \& Pershan, P. S. (1982). Phys. Rev. Lett. 48, 1107-1110.

Blesic, M., Marques, M. H., Plechkova, N. V., Seddon, K. R., Rebelo, L. P. N. \& Lopes, A. (2007). Green Chem. 9, 481-490.

Braslau, A., Pershan, P. S., Swislow, G., Ocko, B. M. \& Als-Nielsen, J. (1988). Phys. Rev. A, 38, 2457-2470.

Broennimann, Ch., Eikenberry, E. F., Henrich, B., Horisberger, R., Huelsen, G., Pohl, E., Schmitt, B., Schulze-Briese, C., Suzuki, M.,
Tomizaki, T., Toyokawa, H. \& Wagner, A. (2006). J. Synchrotron Rad. 13, 120-130.

Buff, F. P., Lovett, R. A. \& Stillinger, F. H. (1965). Phys. Rev. Lett. 15, 621-623.

Daillant, J. \& Gibaud, A. (1999). X-ray and Neutron Reflectivity: Principals and Applications. Paris: Springer.

Fukuto, M., Heilmann, R. K., Pershan, P. S., Badia, A. \& Lennox, R. B. (2004). J. Chem. Phys. 120, 3446-3459.

Fukuto, M., Heilmann, R. K., Pershan, P. S., Griffiths, J. A., Yu, S. M. \& Tirrell, D. A. (1998). Phys. Rev. Lett. 81, 3455-3458.

Fukuto, M., Heilmann, R. K., Pershan, P. S., Yu, S. M., Griffiths, J. A. \& Tirrell, D. A. (1999). J. Chem. Phys. 111, 9761-9777.

Furukawa, Y. \& Matsumoto, N. (2004). Eighth International Conference on Synchrotron Radiation Instrumentation, pp. 12291232.

Kraft, P., Bergamaschi, A., Broennimann, Ch., Dinapoli, R., Eikenberry, E. F., Henrich, B., Johnson, I., Mozzanica, A., Schlepütz, C. M., Willmott, P. R. \& Schmitt, B. (2009). J. Synchrotron Rad. 16, 368-375.

Kudo, T. \& Tanida, H. (2007). Rev. Sci. Instrum. 78, 033104.

Larriba, C., Yoshida, Y. \& Mora, J. F. (2009). J. Phys. Chem. B, 112, 12401-12407.

Li, Z., Zhao, W., Quinn, J., Rafailovich, M. H., Sokolov, J., Lennox, R. B., Eisenberg, A., Wu, X. Z., Kim, M. W., Sinha, S. K. \& Tolan, M. (1995). Langmuir, 11, 4785-4792.

Pershan, P. S. (2000). Colloids Surf. A, 171, 149-157.

Pershan, P. S. (2009). J. Phys. Chem. B, 113, 3639-3646.

Sanyal, M. K., Sinha, S. K., Huang, K. G. \& Ocko, B. M. (1991). Phys. Rev. Lett. 66, 628-631.

Schlossman, M. L., Synal, D., Guan, Y., Meron, M., Shea-McCarthy, G., Huang, Z., Acero, A., Williams, S. M., Rice, S. A. \& Viccaro, P. J. (1997). Rev. Sci. Instrum. 68, 4372-4384.

Schwartz, D. K., Schlossman, M. L., Kawamoto, E. H., Kellogg, G. J., Pershan, P. S. \& Ocko, B. M. (1990). Phys. Rev. A, 41, 5687-5690.

Seddon, K. R. (1997). J. Chem. Technol. Biotechnol. 68, 351-356.

Shpyrko, O., Fukuto, M., Pershan, P., Ocko, B., Kuzmenko, I., Gog, T. \& Deutsch, M. (2004). Phys. Rev. B, 69, 245423.

Shpyrko, O., Huber, P., Grigoriev, A., Pershan, P., Ocko, B., Tostmann, H. \& Deutsch, M. (2003). Phys. Rev. B, 67, 115405.

Sinha, S. K., Sirota, E. B., Garoff, S. \& Stanley, H. B. (1988). Phys. Rev. B, 38, 2297-2311.

Solutskin, E., Ocko, B. M., Taman, L., Kuzmenko, I., Gog, T. \& Deutsch, M. (2005). J. Am. Chem. Soc. 127, 7796-7804.

Terada, Y., Goto, S., Takimoto, N., Takeshita, K., Yamazaki, H., Shimizu, Y., Takahashi, S., Ohashi, H., Furukawa, Y., Matsushita, T., Ohata, T., Ishizawa, Y., Uruga, T., Kitamura, H., Ishikawa, T. \& Hayakawa, S. (2004). AIP Conf. Proc. 705, 376-379.

Tolan, M. (1999). X-ray Scattering from Soft-Matter Thin Films. Berlin: Springer.

Tostmann, H., DiMasi, E., Pershan, P. S., Ocko, B. M., Shpyrko, O. G. \& Deutsch, M. (1999). Phys. Rev. B, 59, 783-791.

Yano, Y. F., Uruga, T., Tanida, H., Toyokawa, H., Terada, Y. \& Takagaki, M. (2007). J. Phys. Conf. Ser. 83, 012024.

Yano, Y. F., Uruga, T., Tanida, H., Toyokawa, H., Terada, Y., Takagaki, M. \& Yamada, H. (2009a). Eur. Phys. Spec. Top. 167, 101-105.

Yano, Y. F., Uruga, T., Tanida, H., Toyokawa, H., Terada, Y., Takagaki, M. \& Yamada, H. (2009b). Langmuir, 25, 32-35.

Yano, Y. F., Uruga, T., Tanida, H., Toyokawa, H., Terada, Y., Takagaki, M. \& Yamada, H. (2009c). Trans. Mater. Res. Soc. Jpn, 34, 631-638.

Yoneda, Y. (1963). Phys. Rev. 131, 2010-2013. 